\title{
$\mathfrak{V} \mathfrak{o} \mathfrak{r} \mathfrak{w} \mathfrak{v} \mathfrak{r} \mathfrak{t}_{\text {. }}$
}

$\widetilde{S}_{\text {eit }}$ Büjđjing's Zeit bat bie Geograpbie yon Arabien in ben (Eompenbien Brade gelegen; eine Durdarbeitung bez feit Niebubr's Zeiten gewonnenen überreiden Stoffez zu einer wiffenidaftididen Darftellung unb Entwidelung ber (Gejammt= verbältniffe biejer merfwürbigen Şalbinfel ift nod nid)t ver=

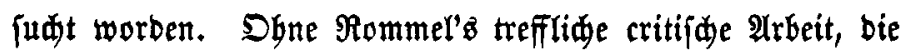
fid) aber nur auf einen (Sommentar zu 2lbulfeba's 2 rabien befäränft, und obne Bergbaus fartographifde 9 arbeit würbe eछ nod) viel fdlimmer bamit in ben geographifden Şanb-

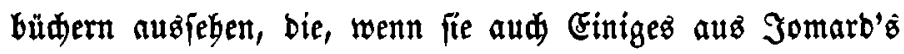
inbaltreiăen 2 rrbeiten über $\mathfrak{A}$ rabien aufgenommen baben, ober

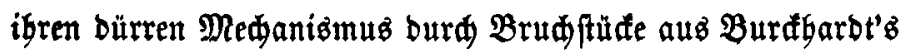
Meifferwerfen ju beleben fudten, ood ein innerlid unzat =

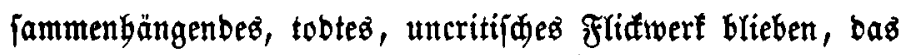
bei weitem bie widtigiten Ergebniffe überging, und fith mit einem nublojen und unfrudtbaren Ballaft von Ramen ogne Jnbalt, alz wäre etwaz babinter, aubitaffirten. $3, \mathfrak{v}$, Sam=

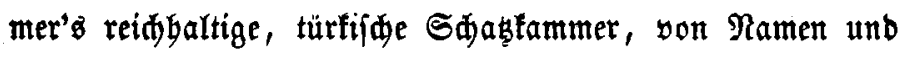

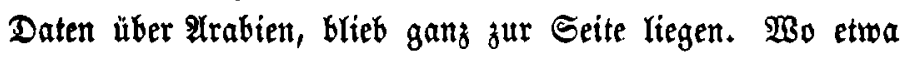

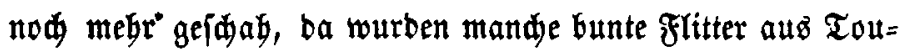
riften willfübrlid berauøgeriffen uno eben to oberflädlid, alz Einiflag in ben magern $3 e t t e l$, eingewebt. Bolf, $\mathfrak{N a}=$ tur unb Ranb blieben neben einanber fteben, bie Ranbegige= 


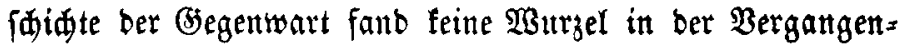
Geit, feinen Spiegel in ber Natur ber Gegenwart. Allez blieb bunfel, ober confus unb ogne inneres Reben, obne Cau= \{alzufammentbantg.

Daz wiffenfdaftide Ergebnip ber befammt= beobahtung eines ganzen Sabrgundertz, fo vieler Stubien unb Betradtungen aużgezeidneter Drientaliften wie Futopten, in ibren, wenn fhon nur partiellen Beftrebun= gen, bod) vft meiftergaften Mittheilungen, alz Reijenbe, $\mathfrak{B} e=$

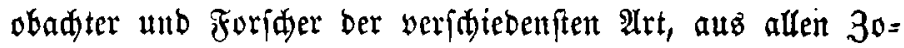
nen, Zeiten, (ulturen, auf arabijđem Boben, in arabifder

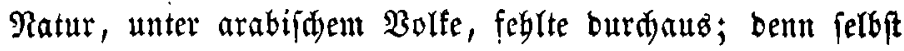
Riebubr's Meifterwerfe waren in bem, waz fie für böberes,

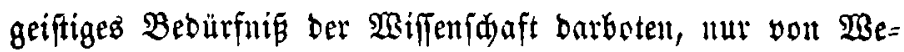
nigen anerfannt, unb voit nod Benigern für ben Fortiðnilt

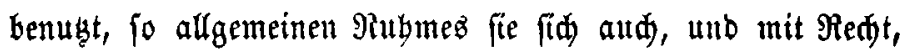
erfreuten.

Und bod ift ein Rand wie $\mathfrak{A} r \mathfrak{a b i c n}$ wol einer burd $=$ britgenbern ernftern (Erforidutug werth; ein Gebiet bes̉ গJIa= teten, bem Areale eines Drittbeits you Europa an 3nbalt

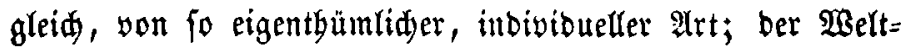
ftellung nady ber Hebergang von 2(fien nad affrifa, beiber Erbtheile Raturen fo daracteriftifd in fid vereitent, wie eigentbümlide, felbftänbige łopulationen berbergent unb ent= wifelnb, benen, zwif Maggreb unb bem duriftitiden Europa, eine ber grofartigften,

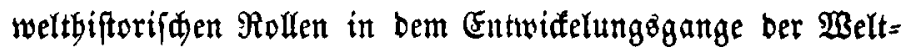
gefđidute übertragen war. Dent bas Gepräge ¡đen Bölferlebenz, bas in Religion, Gejeb, Sprade, Poefie,

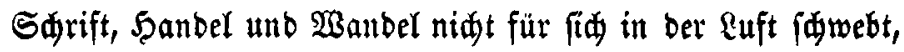
jonbern ber fandesbeimatb, ber Eanbesnatur entwadjen war, 
wurbe, gen अufgang Der Malayenwelt eben fo aufgebrüatt,

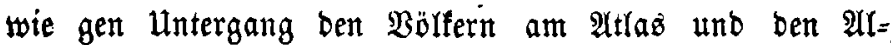
pujaras, wie Denen vom 5 rus, Ełphrat, Tigriz bis̄ zum

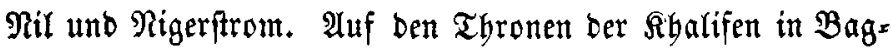
bab, in Gairo, Damast, Sorbova, Sevilfa, Fes, Sajira, Samarfano, Delgi gewann es feinen bönften Ġlanł, auf vielen andern Thronen ber Sultane von $\mathfrak{M a l a f f a}$ biz $\mathfrak{B} \mathfrak{D}=$ fgara, von (Sonftantinopel biz Maroffo, Timbuctu, Darfur und zutrüa biż Sanaa in Jement ift $\mathfrak{e B}$, wie bei ben zuge= Görigen $\mathfrak{B o ̈ l f e r n , ~ n o d ) ~ i n ~ j e i n e r ~ g e i f t e r e r f t a r r e n b e n ~ \Re a w m i r ~ = ~}$

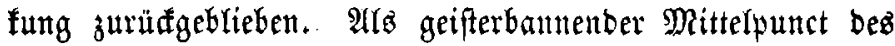
gantzen mobamedanifden Zauberwejenz baben fid) aber in ber

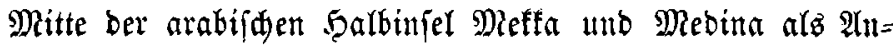

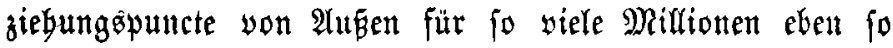
ergalten, wie ìn Snnern Der 5̧albinjel fíd baż patriarda Iijd)e Bebuinenteben ber antifen $\mathfrak{B e l t}$, aub Den 2 hrabamifaen Zeiten bis in bie mobernjten Gerüber in feiter llriprünglid = feit fortzugeftarten sermodte.

Dies̀ fino Erid)einungent in bem fo rätbjelreiden, irbifd)=

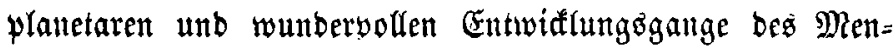
(đ)engeidled)tes, bie fid) voun eltrowäifd = bergebrad)ten, yor= nebmen Stanbpuncte ber Beltbetradtung (ber nod) immer

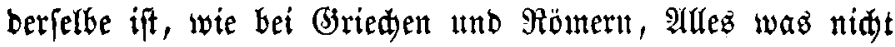
europäifd ift mit bem Ramen barbarifa) jut bejeidunen) nidt burd) bie $\mathfrak{B a r t e : ~ " B B u ̈ f t e " ~ t t t b ~ " \Re o b g e i t " , ~ b i e ~ m a n ~ a u s ~}$

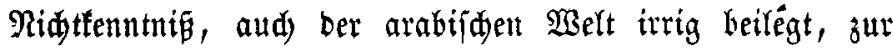
Seite gefdoben und ignoritt werben fönnen unb bürfen. Untb fumal nidjt zu einer Zeit, in weldjer ber Srient, wie ein mädtiger Rieje auz ber langent Erftarrung feines ver= meintliden (s)abes, einer inbaltreid)ern 3ufunft entgegen fid zu erbeben begonnen bat, befien Sdiffjal tein Sterblider in 
ben Sternen za lefen yermag, wie ber einft nod folummernbe

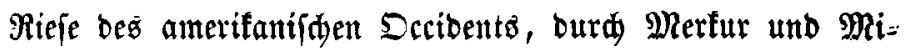
nerva mit bem Derzmeige berilbrt, aus jeiner trümmerreiden

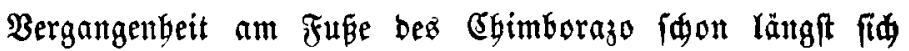

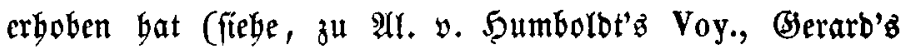
(đ)öntes Titelfupfer: Humanitas, Literae, Fruges) zu gröpe= rem $\mathfrak{D a} \mathfrak{a}$ eint.

Daber muste in einer allgemeinen, vergleidenben, wif = (enfdaftlid)en Betradtung altd biejer ebenbürtigen \$laneten=

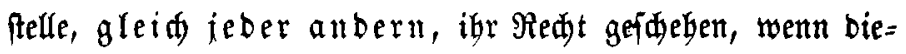
jes audi) nod nidjt nllgemein anerfannt fein follte, weldez

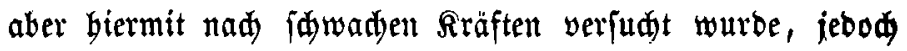
in einer $\mathfrak{A r t}$, bie, bei vielen $\mathfrak{M a ̈ n g e l n , ~ e i n e ~ u n g e m e i n ~ r e i d j e , ~}$ ja wir fönnen fagen, faum geabnete $\mathfrak{A}$ uşbeute gab, nidł Durd eigene $\mathfrak{A}$ rbeit unb Erfinbung etwa, jonbern nur burd gewiffentafteite Beadtung aller berienigen $\mathfrak{B a b r b e i t e n , ~ b i e ~}$ von ben gebirbetiten $\mathfrak{B o r g a ̈ n g e r n ~ u n o ~ Z e i t g e n o f l e n ~ a u f ~ b i e j e m ~}$

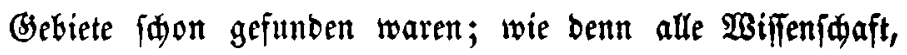
und bie Mgilofopbie felcft, nidits anders ift, ald nur ein $\mathfrak{B} \ell=$ adten bes̃ menfidliden Geiftes und ein 2Auffinden befien, waz,

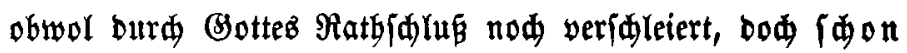
vorbanden war, in ber Matur, in ben menjiliden unb in ben göttfiden Dingen. Nie fann baber bie ftrenge unb

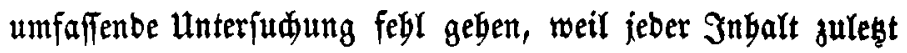
unentlid ift; unb wie follte fie es bier, wo ber vortiegenbe

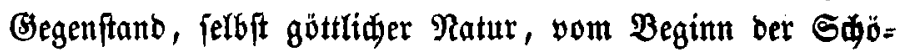
pfung an zur Gefdidte ber Menfdyeit in ibrer yar s monifden Entwialung mit bem Erbbarl gebörte, zur Ermittlung ber Culturftufe mit ber igr zugetwiefenen Stelfe

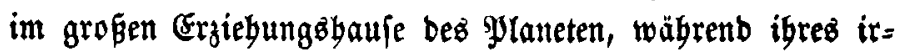
bifiden Dafeinz. 
Einen, gegent frübete, furggefaßtere Atbriffe Arabiens,

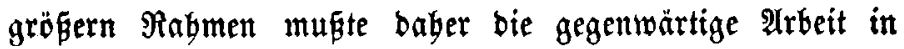

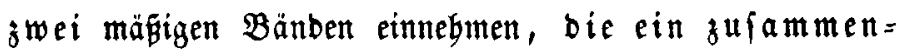
băngenbeb, für fid abgefdloffenes (banze bilben, weil auz Der compacten, in fid zufammenbängenbern Maffe अfienz beffen weft wärt immer mebr unb mebr fortidyret= tenbe (Glieberung aud wirflid Sonberbetradtungen bieier einzelnen Grieber (wie Arabien, Syrien, Afra mi= nor, Raufajuş) niđt nur mögliđ, fonbern, alö eigne fleine Belten für fíd, notbwendig madte. Daber benn zu ber (6) ruppirung von $D$ ft $=\mathfrak{A}$ fien in vorigem (Bb. II bio VI),

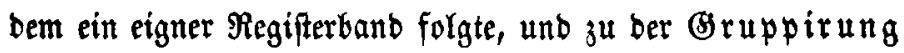
von $\mathfrak{B}$ eft $=\mathfrak{A}$ fien (Bb. VII bis XI), befien fünf $\mathfrak{B a ̈ n b e n}$ unmittelbar ein zweiter Regifterbanb folgen wirb, aud bie

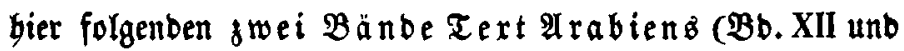

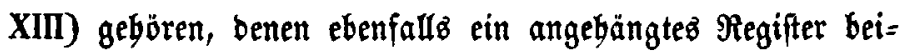
gegeben jein woirb. $3 \mathfrak{u}$ ibrem Gejammtyerfänbni tạn, bei ber grofen unvolftommenbeit bişberiger Generalfarten, ein eignes 5̧eft "ঐartenmaterial zu Arabien" ausigege= ben werben, beர̄en gewiffenbafte Mebaction, na由 ben uns von ben verífiebenften Seiten bargebotnen, fum Theil fonft nod) unbenuß̧ten banbidjriftlidjen Suellen, wir bem raftlojen Eifer unjerz jüngern Freunbeb̆, bem Ricutn. 3immermann som Seneralfiabe, verbanfen, ber fich fdon in fo vieler Şin= fidt um fortbilbung ber Erbfunde im $\mathfrak{I} \mathfrak{n}=$ unb $\mathfrak{A}$ uslanbe nidt unbeadtet gebliebene Berbienjte erworben bat. Uniferm jüngern Freunbe $\mathfrak{R}$ iepert verbanfen wir bie jorgfältige

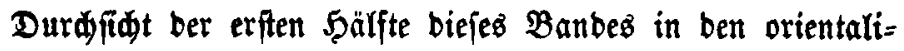
iđen Ramen beim Druffe, bevor berfelbe nad $\mathfrak{B}$ Beimar über= fiebelte, und $\mathfrak{S r}$. G. Fr. S. Mülller nidt nur bie forgfältigfte unterftüß̧ung bei ber Correctur bes̀ ganzen Banbes, fonbern 


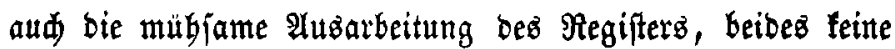
geringen Bemühutngen, bie Daber aud wol bie Radjfidt ber Sienner in 2 njprud negmen bürften.

Die Bergleiduung ber Angaten ber vier aubgezetadtete= ftent orientalijđen (Geograpben über 9 (rabien, mit weld)er bie= fer erfte $\mathfrak{B a n b}$ begimut, war ein neugemagter $\mathfrak{B e r}$ ud), ber

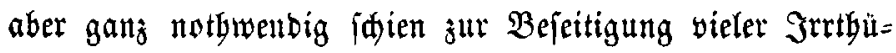
mer unb um nur bie Begenwart verfteben ju lernen, bie aud im geograptifiden, wie im biftorifden, auf biejem $\mathfrak{B} \mathfrak{D}$ ben unt unter biejem Bolfe faft nur auf ber $\mathfrak{B e r g a n g e n t =}$

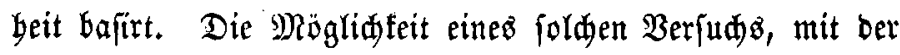

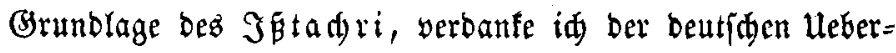
feksung meines jüngern Freundes A. D. Morbtmann, jekst Gefdäftzträget und Gejandtel ber Freeten Şauleeftäbte an ber Soben Pyorte in Sonftantintupel, bie feitbem autd offentlid

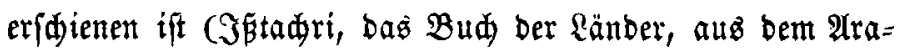

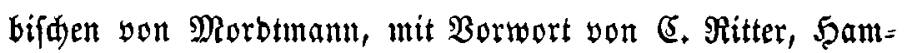
$\mathfrak{b u r g}, 1845,4$.), bie aber nur erit im Micr., nidjt im Druf́,

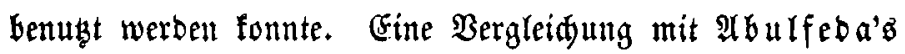
youftänbigen Texte wurbe mir butd) meinen fo zuvorfom=

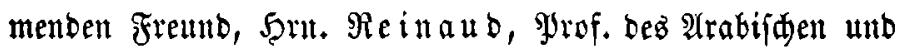

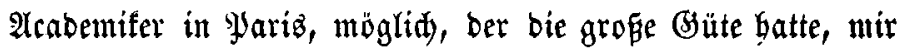
feine franzöfif

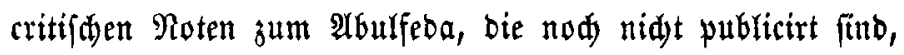

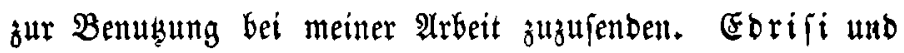
(É $\mathfrak{n} \mathfrak{B} \mathfrak{a} \mathfrak{a t} \mathfrak{t a}$ waren

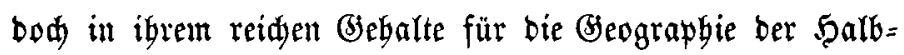

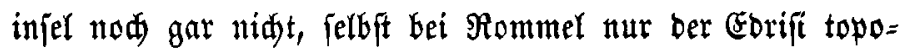
grapbifa, benukgt. Den aus foldem Berjudje bervorgegan=

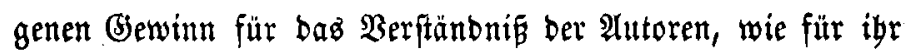
Ranb unb Bolf, bleibe been $\mathfrak{H}$ rtbeil ber Senner übertaffen. 


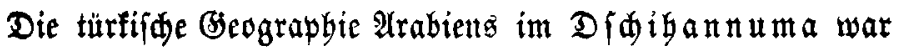
burd) unfern bodueregrten Gönner uno Freunt $\mathfrak{j}, \mathfrak{v}, \mathfrak{5} \mathfrak{a m}=$ mer idon to vortreffitid in igrem rexicalifaen Ueberreidthum georbnet, Daß́ wir auf ifren volftänoigen fluk nur binzumeifen brautden, wo ez für unire $\mathfrak{A r b e i t ~ n o ̈ t b i g ~ i đ i e n . ~}$

Die reide Fülle ganz netent Materials, wie von $S_{a b}=$

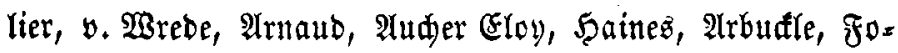

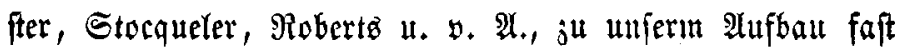

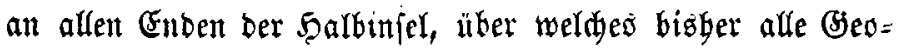
graphien gejdrwiegen, baben wir hier nidst aufäzäblen, ba baffelbe überall im $\mathfrak{B e r f e}$ feiner Dutle nad genau bejeid)= net ift. Sobwol unir unz feinesiweg $\mathfrak{s}$ mit bem Titel einer voll ftänbigen Geograybie von $\mathfrak{A r a b i e n ~ b r u ̈ f t e n t ~ w o l l e n , ~ b a ~}$ ia $\mathfrak{B i e l e b ~ n o d ~ T e r r a ~ i n c o g n i t a , ~ u n d ~ w o n ~ b e e n ~ f d j o n ~ B e f a m t = ~}$ ten jelbit gar Mandjeb, nad) Ramen und Saden, bajelbjt für eine Dereinftige lexicalijøe Einregiftrinung übrig bleibt, mit ber wir Gier nidib fa thut baben, fo glaubent wir bod

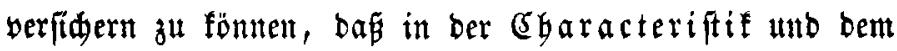

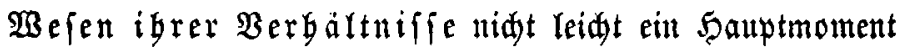
yermift werben bürfte.

Da wir überall Dem immerften Gedanfengange in ber Beididte bes Bolfz, feiner Jyolitif, friner Religion, feiner Sitten und Deren mannidjaltigftent Reflexen und Manifeíta= tionen in Sprade unb Siteratur, wie feinen treueften uno

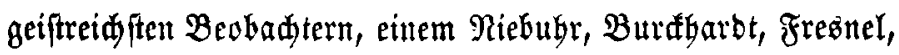

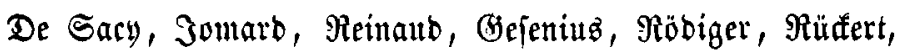
Beil, Enwald t. $\mathscr{A}$., żu folgen utb bie eigentgümlide $\mathfrak{N a}=$ tur beż Ranbes wie feiner Drgantismen z̆u erfennen bemübt

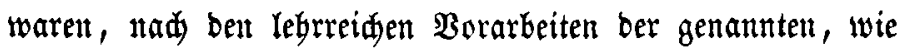
eines Forstäl, Seetsen, Egrenberg, Botta, Rüpwedl, Sdjim= per, $\mathfrak{B e l l f t e b}$, Şulton, Eruttenben, Malcolm, $\mathfrak{B i c}$. Balentia, 


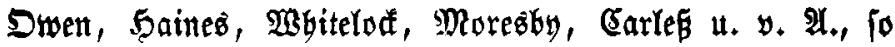
trat mun bie vermeintlidie "Wülte" an fo vielen Stellen alö cin wirflid) reid begabte und bevölferte Ranto, bie eben to für blos "roh und barbarifø gebaltne wilde Bolfżmaffe,"

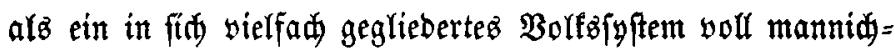
faltig gefonberter Jinbivioualtiäten und Entwif́etungen zut bent

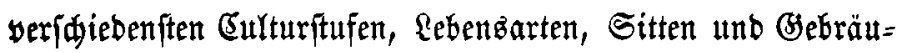
den berwor, wie fie ber Bang ber Bölfergeididte, bie bier

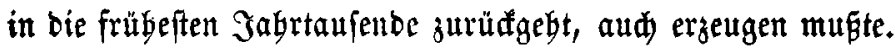
Das Rand zeigte fid reid) an biftorifden 2 (ntlängent in allent Riđtungen ber älteften Bölfergeididten, von benten $\mathfrak{e}$, wenn

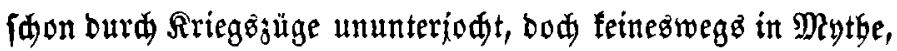
Gentealogien, Sprad)en, 5̧iftorien, Eulturen, Eiteratur, Joeen, fo gejonbert und ijolirt ober zurüă geblieben, ba man ím allein nur etwa antifen $\mathfrak{B}$ eltbantel und Bertegr, zur 3eit

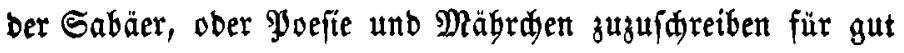
fanb, alle anbern 3 weige ber Entwiflung aber igm oft von vorn berein verjagen zu mùffen wäbnte.

Die altejte Periobe bat nun idon igren nod uneridäpf= ten Reidtbum an überbauernben Monumenten an allen Ge= ftaben und an bielen Rocalitäten Des̃ Snnern Dargeboten, bie burd igre zablreiden antifen $\mathfrak{I n f}$ criptionen auz einer ver= [đwutbenen Gulturperiobe bereinft efen fo, um bie Metropolis Regia Sabaeorum bas Dunfel ber Bergangenheit erbellen werben, wie bie Denfmale zu Theben am Rit, zu Babylon am Eupbrat, zu Rinivel am Tigriz, wie bie am కnbuz unb (Bangez, in $\mathfrak{Y}$ ucatan $\mathfrak{P a l e n q u e , ~ u n o ~ i n ~ n o d ) ~ a n b e r n ~ u n b u r d ) = ~}$ foridten amerifanifden $\mathfrak{u}$ rwärbern.

Die jüngfte 3eit ber äguptifden Rriegg̈periobe Mobam= med Artiz gegent bie $\mathfrak{B a b a b i}$ unb ber britifaen $\mathfrak{P}_{0}$ litif in Arabien bat, nod mebr alò bie lange vorbergegangene Frie= 


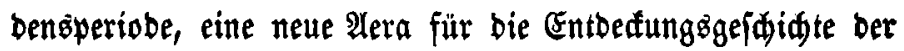

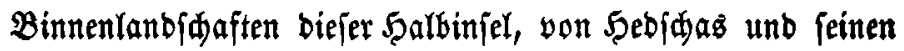
Bergfetten, yon Rebided, von Babrein und ben Siraten= füften, von 2rjor, jelbit von Theilen Jemenb, von aben uno Dman, Gerbeigefübrt, Deren Rejultate wir, Durd Die Zeitum= ftänbe megrfađ begüniftigt, zum erftenmale in iłrem erfolg=

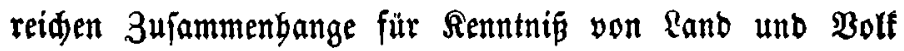
Gier für bie Erbfunbe frudttbringend vorfübren fonnten.

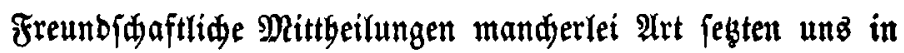
Stand, bie $\mathfrak{B i f f e n f d a f t ~ b u r d ~} \mathfrak{B e r t i l g u n g ~ m a n d e r ~ S r r t g u ̈ m e r ~}$

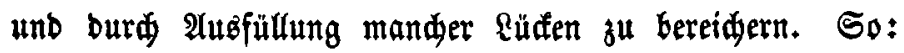

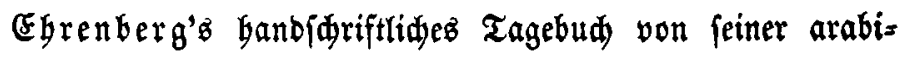

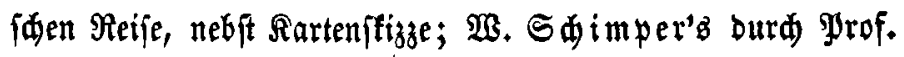

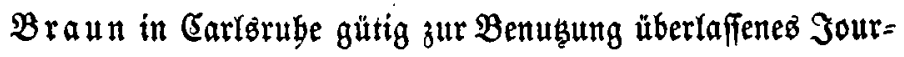
nal bes Routier von Didibba nad Taif. Eben fo bez öjtreidifden Gejanbten Graf $\mathfrak{y}$. Stürmer Uebergabe lebr= reidjer (Conjulatäberidte über bie Rriege in $\mathfrak{A} j$ yr, bei unjerm 2ufentbalte in Eonftantinopel; ferner bie vielen förbernden Beiträge, bie wir wäbrenb unjerz leşten $\mathfrak{A}$ ufentbaltez in

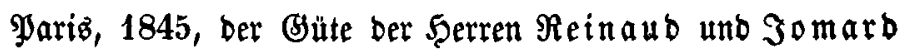
verbanfen; auథ bie Geftattung einer Gopie bes Ganbifrift= liđen febr iđäß̨baren neuen Rartenentwurfes ber Sngenieure

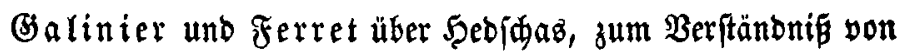

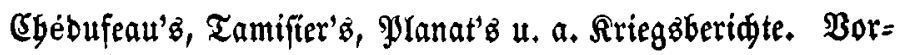

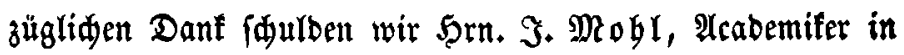

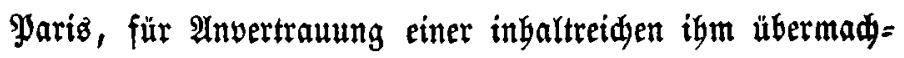
ten (Sorrefponbenz bes gelebrten und geiftreiden Spradfor=

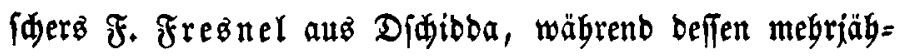

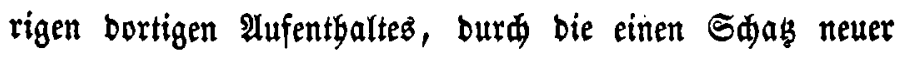
Foridungen und Beobadtungen für unfere Biffenjđaft ge= wonnen zu baben, wir bier zum vorauz anerfennen. Die 
श्Anwenbung, Die wit von allen biefen Baben, oft nur ans fübrungg̊weife, um zu ueten Foridungen an Drt und Stelfe aufzuforbern unb auf vielez biobber Ueberiebene aufmerffam zu mad)en, wie wou fo mandent andern, für ben Fortiantitt ber (Erbfunbe gemad)t baben, möge jenen Gebern genebm. Fein, und mebr als $\mathfrak{B}$ orte unjere Berbintblidfeit bafür bezeu= gen, fowol in biejem erften $\mathfrak{B a n d e}$ als in unmittelbar nadfolgenden zweiten, bem aud ein angang über geo= grapbiðde Berbreitung fipgären einiger für Ârabien

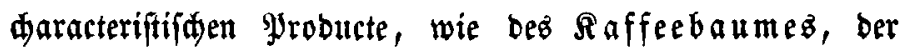
Dattelpalme, bes $\mathfrak{A} a m e e l s$ u. $\mathfrak{a}_{*} ;$ nebjt Regifter und arabifđem Rartenbeft beigefügt fein wirb.

Berrin, am 26. März 1846.

\section{હ. Ritter.}

